\title{
The Solution and Numerical Simulation of Several Three Dimensional Convection Diffusion Equations
}

\author{
Zhengwei Dong, Ji,ang Zhang \\ School of Electrical Engineering, Shan Dong University, Jinan, 250002, China \\ 603936398@hotmail.com
}

Keywords: three dimensional convection diffusion equation; fourier transform; numerical solution of inte-gral; The simulation with MATLAB

\begin{abstract}
In this paper, the instantaneous point source diffusion model, the continuous time source diffusion time-varying model and the continuous time arbitrary shape source diffusion time-varying model in convection-diffusion equation are studied. First, by establishing the appropriate model assumptions, under certain initial conditions, the analytical solution of instantaneous point source diffusion model is obtained by Fourier transform. Using this result can get the solution of the source diffusion model at the continuous time point by integrating the result in time. The volume of the source shape and the integral of the time are obtained, and the solution of the diffusion model with arbitrary shape in continuous time is obtained, a series of graphs (chamfer, gradient, contour, etc.) describing the diffusion characteristics were drawn; and the solution images of each model were analyzed and compared.
\end{abstract}

\begin{abstract}
This article studies the instantaneous point source diffusion time-varying model, the continuous time point source diffusion time-varying model and the continuous time shape source diffusion time-varying model. A proper model assumption is established firstly. Under the given initial conditions, the analytical solution of instantaneous point source diffusion model is obtained with Fourier transform. The solution of the continuous time point source diffusion time-varying model is obtained with the integral in time domain. And the solution of the continuous time shape source diffusion time-varying model is obtained with the triple integral in the shape of the source and then the integral in time domain. Then, the numerical simulation of these models above is given with the help of MATLAB, drawing a series of images which describe the characteristics of the diffusion (The sectional drawing, gradient map, contour map and so on). The analysis and comparison of these images are given.
\end{abstract}

\section{Introduction}

Air pollution and water pollution are increasingly serious, threatening the survival of mankind, how to better control pollution, people pay more and more attention. The diffusion of air pollutants and water pollutants in the environment follows the same laws of mathematical physics: the convection-diffusion equation is a large class of partial differential equations that can be used to quantitatively measure the spatial and temporal distribution of pollutants in the environment and help people to efficiently and effectively Managing pollution rationally has also played a huge role in areas such as thermal and electromagnetism.

Convection diffusion equation has a very high theoretical value and broad application background. Different application backgrounds lead to different boundary conditions and initial conditions, and the result and form of solution are also different. The vast majority of difficult to get analytic solution needs to use computer numerical simulation. Under the reasonable assumption, instantaneous point source convection diffusion model, continuous point source convection diffusion model and continuous arbitrary shape source convection diffusion model are established. In a class of initial conditions, you can get beautiful analytical results. At the same time, the algorithm of numerical solution of equations is given. The numerical simulation is carried out by 
means of MATLAB, which realizes the data processing and realizes the mathematics and physics essence of convection and diffusion more three-dimensionally and vividly.

\section{Model Establishment}

In order to reveal the mathematical physics of convection-diffusion equations, we must create a proper physical background. Here we study the problem of convection and diffusion of pollutants in the atmosphere.

\section{Put Forward Model}

(1) Instantaneous point source diffusion model. The source and scale of the initial momentary release are negligible compared to the spatial scale considered. The mathematic model is set up to study the spatial and temporal distribution of pollutant concentration in the atmospheric space near the source, and the variation law within a certain range can be investigated by defining the spatial scale. Assuming that the nearby space is a cuboids space "long is $L$, wide is $W$, high is $H^{\prime \prime}$, the pollution source can be set at the center of the cuboids.

(2) Continuous point source diffusion model. The sources and scales released for continuous time are negligible compared to the spatial scales considered. The establishment of mathematical model to investigate the spatial and temporal distribution of pollutants in the atmospheric space near the source spatial and temporal distribution of spatial scale set up as above.

(3) Continuous $\Sigma$ Diffusion Model. The source of continuous release, with shape $\Sigma$, can not be ignored. The establishment of mathematical model to investigate the spatial and temporal distribution of pollutants in the atmospheric space near the source spatial and temporal distribution of spatial scale set up as above.

\section{Model Assumptions and Symbolic Conventions}

The velocity $\left(V_{x}, V_{y}, V_{z}\right)$ of the air in the $X, Y, Z$ direction is constant during the investigated period, that is, the weather is relatively stable. The pollutant concentration $u(x, y, z, t)$ at the space point $(x, y, z)$ and the diffusion coefficient $(D x, D y, D z)$ in the $X, Y, Z$ direction at $t$ time are constant over the investigated time period, That is, the diffusion process is relatively stable; the atmosphere has the ability of self-purification, and the unit concentration of pollutant concentration per unit time in the $(x, y, z)$ is proportional to $u(x, y, z, t)$ at this time, proportionality coefficient is $f$; the air can flow randomly and disorderly.

\section{Model Establishment}

The following establishes the mathematical model of convection diffusion equation, the study of space is divided into micro-elements $(d x d y d z)$, and each micro-element has material inflow and outflow every moment, respectively, the positive axis of the material diffusion as the positive direction, by the law of diffusion:

$$
\vec{q}=-D \nabla u
$$

In which $D$ is the second-order tensor, $q$ is the diffusion flow intensity (mass per unit time through the unit cross-section), and the component expression is:

$$
q_{x}=-D_{x} \frac{\partial u}{\partial x}, \quad q_{y}=-D_{y} \frac{\partial u}{\partial y}, \quad q_{z}=-D_{z} \frac{\partial u}{\partial z}
$$

Take the $X$ direction to examine, the diffusion flow from $x$ into, out from $x+d x$, at the unit time, the micro-elements in the $X$ direction of the diffusion flow increase is:

$$
\left(\left.q_{x}\right|_{x}-\left.q_{x}\right|_{x+d x}\right) d y d z=\left[-\left.D_{x} \frac{\partial u}{\partial x}\right|_{x}-\left(-\left.D_{x} \frac{\partial u}{\partial x}\right|_{x+d x}\right)\right] d y d z=\frac{\partial}{\partial x}\left(D_{x} \frac{\partial u}{\partial x}\right) d x d y d z
$$

In the same the increment in the $Y, Z$ direction is respectively:

$$
\frac{\partial}{\partial y}\left(D_{y} \frac{\partial u}{\partial y}\right) d x d y d z \frac{\partial}{\partial z}\left(D_{z} \frac{\partial u}{\partial z}\right) d x d y d z
$$

Then from 0 to $t$ moment, because of the concentration difference diffusion into the study space the amount of material as follows: 


$$
\int_{0}^{t}\left[\iiint_{v}\left(\frac{\partial}{\partial x}\left(D_{x} \frac{\partial u}{\partial x}\right)+\frac{\partial}{\partial y}\left(D_{y} \frac{\partial u}{\partial y}\right)+\frac{\partial}{\partial z}\left(D_{z} \frac{\partial u}{\partial z}\right)\right) d x d y d z\right] d t
$$

The air flow velocity of air point is $\left(V_{x}, V_{y}, V_{z}\right)$, respectively, the $X, Y, Z$ axis is positive direction. Take the $X$ direction inspection, pollutants with air inflow from $x$, at the outflow from $x+d x$, in units of time, the outflow of micro-elements in the $X$ direction is:

$$
\left(\left.u\right|_{x+d x}-\left.u\right|_{x}\right) V_{x} d y d z=\frac{\partial u}{\partial x} V_{x} d x d y d z
$$

Similarly, the outflow of the $Y, Z$ direction is respectively:

$$
\frac{\partial u}{\partial y} V_{y} d x d y d z, \frac{\partial u}{\partial z} V_{z} d x d y d z
$$

From 0 to $t$, the amount of material leaving the study space with the flow of air is:

$$
\int_{0}^{t}\left[\iiint_{v}\left(\frac{\partial u}{\partial x} V_{x}+\frac{\partial u}{\partial y} V_{y}+\frac{\partial u}{\partial z} V_{z}\right) d x d y d z\right] d t
$$

Due to the self-purification of air, the amount of material reduced from 0 to $t$ is:

$$
\int_{0}^{t}\left[\iiint_{v} f_{u}(x, y, z, t) d x d y d z\right] d t
$$

The inflow minus the outflow is the material increase over this period, expressed as:

$$
\int_{0}^{t}\left[\iiint_{v} \frac{\partial u}{\partial t} d x d y d z\right] d t
$$

Meet $(4)=(1)-(2)-(3)$, and remove the integral number, too:

$$
\frac{\partial u}{\partial t}=\left(\frac{\partial}{\partial x}\left(D_{x} \frac{\partial u}{\partial x}\right)+\frac{\partial}{\partial y}\left(D_{y} \frac{\partial u}{\partial y}\right)+\frac{\partial}{\partial z}\left(D_{z} \frac{\partial u}{\partial z}\right)\right)-\left(\frac{\partial u}{\partial x} V_{x}+\frac{\partial u}{\partial y} V_{y}+\frac{\partial u}{\partial z} V_{z}\right)-f u
$$

The instantaneous point source diffusion model is the most basic model. Other types of sources can be represented by instantaneous point sources, expressed by integrals in time or space. When the point source is located $\left(x_{0}, y_{0}, z_{0}\right)$, the initial conditions are:

$$
u(x, y, z, 0)=N \delta\left(x-x_{0}, y-y_{0}, z-z_{0}\right)
$$

$N$ is the material put into the moment of $0, \delta(x, y, z)$ is the three-dimensional impact function units to meet:

$$
\int_{-\infty}^{\infty} \int_{-\infty}^{\infty} \int_{-\infty}^{\infty} \delta(x, y, z) d x d y d z=1
$$

\section{Model Solution}

Use Fourier transform to solve (5), the initial condition is (6):

$$
\left\{\begin{array}{l}
\frac{\partial u}{\partial t}=\left(\frac{\partial}{\partial x}\left(D_{x} \frac{\partial u}{\partial x}\right)+\frac{\partial}{\partial y}\left(D_{y} \frac{\partial u}{\partial y}\right)+\frac{\partial}{\partial z}\left(D_{z} \frac{\partial u}{\partial z}\right)\right)-\left(\frac{\partial u}{\partial x} V_{x}+\frac{\partial u}{\partial y} V_{y}+\frac{\partial u}{\partial z} V_{z}\right)-f u \\
u(x, y, z, 0)=N \delta\left(x-x_{0}, y-y_{0}, z-z_{0}\right)
\end{array}\right.
$$

Retain $t$, use the Fourier transform for the equations and initial conditions $x, y, z$, and (7) becomes:

$$
\left\{\begin{array}{l}
\frac{d U\left(\omega_{x}, \omega_{y}, \omega_{z}, t\right)}{d t}=\left(\left(\left(i \omega_{x}\right)^{2} D_{x}+\left(i \omega_{y}\right)^{2} D_{y}+\left(i \omega_{z}\right)^{2} D_{z}\right)-i \omega_{x} V_{x}-i \omega_{y} V_{y}-i \omega_{z} V_{z}-f\right) U\left(\omega_{x}, \omega_{y}, \omega_{z}, t\right) \\
U(x, y, z, 0)=N e^{-\left(i \omega_{x} x_{0}+i \omega_{y} y_{0}+i \omega_{z} z_{0}\right)}
\end{array}\right.
$$


First-order linear differential equation of the type $U$, special solution is:

$$
U\left(\omega_{x}, \omega_{y}, \omega_{z}, t\right)=N e^{-\left(i \omega_{x} x_{0}+i \omega_{y} y_{0}+i \omega_{z} z_{0}\right)-\left(\omega_{x}^{2} D x+\omega_{y}^{2} D y+\omega_{z}^{2} D z+i \omega_{x} V_{x}+i \omega_{y} V_{y}+i \omega_{z} V_{z}+f\right) t}
$$

$U$ inverse Fourier transform, $u(x, y, z, t)=$

$$
\begin{aligned}
& =\frac{N}{(2 \pi)^{3}} \int_{-\infty}^{\infty} \int_{-\infty}^{\infty} \int_{-\infty}^{\infty} e^{-\left(i \omega_{x} x_{0}+i \omega_{y} y_{0}+i \omega_{z} z_{0}\right)-\left(\omega_{x}^{2} D x+\omega_{y}^{2} D y+\omega_{z}^{2} D z+i \omega_{x} V_{x}+i \omega_{y} V_{y}+i \omega_{z} V_{z}+f\right) t+i \omega_{x} x+i \omega_{y} y+i \omega_{z} z} d \omega_{x} d \omega_{y} d \omega_{z} \\
& =\frac{N}{(2 \pi)^{3}} \int_{-\infty}^{\infty} \int_{-\infty}^{\infty} \int_{-\infty}^{\infty} e^{-\left(\omega_{x}^{2} D x+\omega_{y}^{2} D y+\omega_{z}^{2} D z+i \omega_{x} V_{x}+i \omega_{y} V_{y}+i \omega_{z} V_{z}+f\right) t+i \omega_{x}\left(x-x_{0}\right)+i \omega_{y}\left(y-y_{0}\right)+i \omega_{z}\left(z-z_{0}\right)} d \omega_{x} d \omega_{y} d \omega_{z}
\end{aligned}
$$

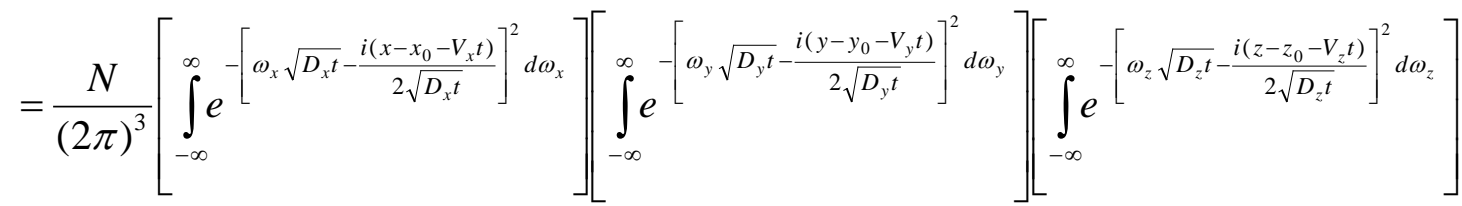

$$
\begin{aligned}
& =\times\left[e^{-\left[\frac{\left(x-x_{0}-V_{x} t\right)}{2 \sqrt{D_{x} t}}\right]^{2}-\left[\frac{\left(y-y_{0}-V_{y} t\right)}{2 \sqrt{D_{y} t}}\right]^{2}-\left[\frac{\left(z-z_{0}-V_{z} t\right)}{2 \sqrt{D_{z} t}}\right]^{2}-f t}\right] \\
& \omega_{x} \sqrt{D_{x} t}-\frac{i\left(x-x_{0}-V_{x} t\right)}{2 \sqrt{D_{x} t}}=\alpha_{x}, \omega_{y} \sqrt{D_{y} t}-\frac{i\left(y-y_{0}-V_{y} t\right)}{2 \sqrt{D_{y} t}}=\alpha_{y}, \omega_{z} \sqrt{D_{z} t} t \frac{i\left(z-z_{0}-V_{z} t\right)}{2 \sqrt{D_{z} t}}=\alpha \\
& \frac{N}{(2 \pi)^{3}}\left[\int_{-\infty}^{\infty} e^{-\alpha_{x}^{2} d \frac{\alpha_{x}}{\sqrt{D_{x} t}}}\right]\left[\int_{-\infty}^{\infty} e^{-\alpha_{y}^{2} d \frac{\alpha_{y}}{\sqrt{D_{y} t}}}\right]\left[\int_{-\infty}^{\infty} e^{-\alpha_{z}^{2} d \frac{\alpha_{z}}{\sqrt{D_{z} t}}}\right] \times\left[e^{-\left[\frac{\left(x-x_{0}-V_{x} t\right)}{2 \sqrt{D_{x} t}}\right]^{2}-\left[\frac{\left(y-y_{0}-V_{y} t\right)}{2 \sqrt{D_{y} t}}\right]^{2}-\left[\frac{\left(z-z_{0}-V_{z} t\right)}{2 \sqrt{D_{z} t}}\right]^{2}-f t}\right]
\end{aligned}
$$$$
=\frac{N}{8(\pi t)^{3 / 2} \sqrt{D_{x} D_{y} D_{z}}} e^{-\left[\frac{\left(x-x_{0}-V_{x} t\right)}{2 \sqrt{D_{x} t}}\right]^{2}-\left[\frac{\left(y-y_{0}-V_{y} t\right)}{2 \sqrt{D_{y} t}}\right]^{2}-\left[\frac{\left(z-z_{0}-V_{z} t\right)}{2 \sqrt{D_{z} t}}\right]^{2}-f t}
$$

At this point, we get the $\left(x_{0}, y_{0}, z_{0}\right)$ point source with instantaneous amount $N$ at zero position and the analytic formula of pollutant concentration around the space point $(x, y, z)$ at $t$ time.

It can be promoted as follows:

(1) Instantaneous point sources are superposed in space and other types of instantaneous sources can be found, spatiotemporal distribution of concentration in their space. Let the shape of the source be $\Sigma$, the spatiotemporal function $u(x, y, z, t)$ of the concentration distribution around the instantaneous source $\Sigma$ :

$$
\iiint\left[\frac{N}{8(\pi t)^{3 / 2} \sqrt{D_{x} D_{y} D_{z}}} e^{-\left[\frac{\left(x-x_{0}-V_{x} t\right)}{2 \sqrt{D_{x} t}}\right]^{2}-\left[\frac{\left(y-y_{0}-V_{y} t\right)}{2 \sqrt{D_{y} t}}\right]^{2}-\left[\frac{\left(z-z_{0}-V_{z} t\right)}{2 \sqrt{D_{z} t}}\right]^{2}-f t}\right] d x_{0} d y_{0} d z_{0}
$$

At this moment, $N$ is the quality of the pollutants put on $\left(x_{0}, y_{0}, z_{0}\right)$ at 0 moments.

(2) The instantaneous point source is superposed on time 0 to $t$ and the spatiotemporal function $u(x, y, z, t)$ of the concentration distribution around the point source at the continuous point in time can be obtained:

$$
\int_{0}^{t}\left[\frac{N}{8(\pi t)^{3 / 2} \sqrt{D_{x} D_{y} D_{z}}} e^{-\left[\frac{\left(x-x_{0}-V_{x} t\right)}{2 \sqrt{D_{x} t}}\right]^{2}-\left[\frac{\left(y-y_{0}-V_{y} t\right)}{2 \sqrt{D_{y} t}}\right]^{2}-\left[\frac{\left(z-z_{0}-V_{z} t\right)}{2 \sqrt{D_{z} t}}\right]^{2}-f t}\right] d t
$$

At this moment, $N$ is the quantity of pollutants released at the unit time. The same is below. 
(3) Superimposing on the instantaneous $\Sigma$ source from 0 to $t$, we obtain the spatiotemporal function $u(x, y, z, t)$ of the concentration distribution around the source of continuous time at $t$ time:

$$
\int_{0}^{t}\left\{\iiint\left[\frac{N}{8(\pi t)^{3 / 2} \sqrt{D_{x} D_{y} D_{z}}} e^{-\left[\frac{\left(x-x_{0}-V_{x} t\right)}{2 \sqrt{D_{x} t}}\right]^{2}-\left[\frac{\left(y-y_{0}-V_{y} t\right)}{2 \sqrt{D_{y} t}}\right]^{2}-\left[\frac{\left(z-z_{0}-V_{z} t\right)}{2 \sqrt{D_{z} t}}\right]^{2}-f t}\right] d x_{0} d y_{0} d z_{0}\right\} d t
$$

In (2) and (3), since $\Sigma$ is a bounded space and the integral is the natural logarithm of the flat type, we can not find the analytical solution. We can use MATLAB software to do the numerical simulation.

\section{Numerical Simulation based on MATLAB}

First of all, the distribution functions in the various parameters assignment:

$$
\begin{gathered}
L=600 \mathrm{~m}, W=400 \mathrm{~m}, H=100 \mathrm{~m}, D_{x}=5 \mathrm{~m}^{2} / \mathrm{s}, D_{y}=6 \mathrm{~m}^{2} / \mathrm{s}, D_{z}=20 \mathrm{~m}^{2} / \mathrm{s} \\
V_{x}=1.5 \mathrm{~m} / \mathrm{s}, V_{y}=1.6 \mathrm{~m} / \mathrm{s}, V_{z}=1.4 \mathrm{~m} / \mathrm{s}, N=30000 \mathrm{~g}, f=0.5 \times 10^{-3} \mathrm{~s}^{-1}
\end{gathered}
$$

It can be set according to the actual situation.

It is necessary to simulate the three cases separately, subject to article length limitation. In this paper, only the numerical simulation of source diffusion at instantaneous point is given.

According to Eq. (8), we simulated the concentration distribution curve of $X O Y$ plane, the concentration distribution contour of $X O Y$ plane, the concentration profile of different sections and the concentration gradient of the whole space at $t$ time convection diffusion process.

Fig. 1 shows the concentration profile on XOY after $20 \mathrm{~s}$ and the Z-axis data shows the concentration. Fig. 2 is the contours of the surface of Fig. 1, each contours have corresponding concentration values.

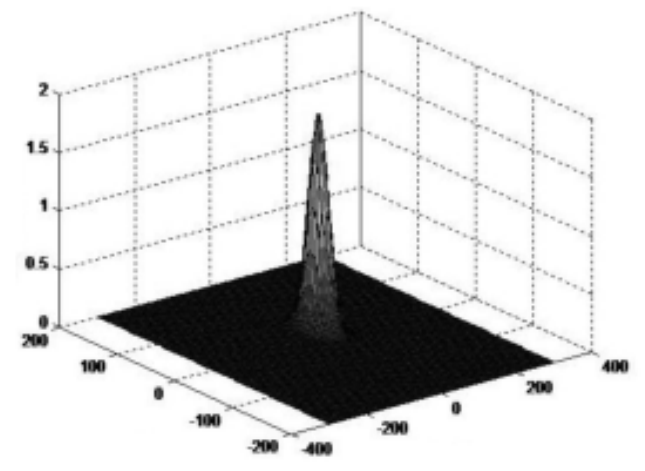

Fig. 1

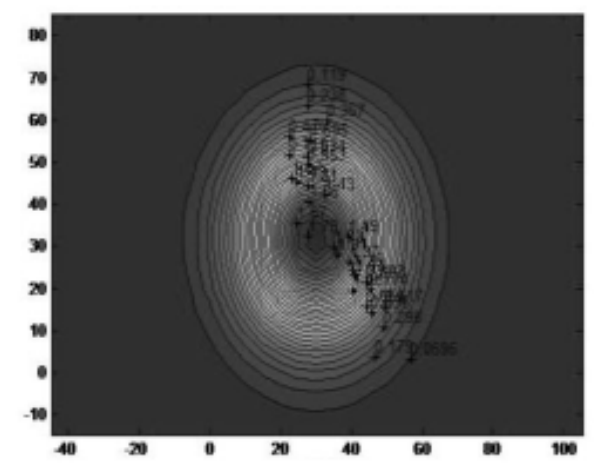

Fig. 2

Fig. 3, fig. 4, fig. 5, Fig. 6, respectively shows when $t=t, 10,20,100 s$, while the concentration distribution in the plane $z=8,-2,4$, can be seen from the figure, with the passage of time, on the section of the pollution source area gradually expanded (part of the outer black concentration is zero, said this part, but not the diffusion peak diffusion section) the center is gradually reduced, and the diffusion center to edge migration. According to the numerical results, the positions and concentration amplitudes of the diffusion centers at any cross section can be known.

Fig. 7 shows when the concentration gradient vector $t=20 \mathrm{~s}$ diagram of the diffusion of the instantaneous point source at the moment, and the diffusion trend is columnar distribution. You can see the fastest change of concentration at this point in time.

\section{Model Evaluation and Improvement}

The paper studied the mechanism of three dimensional convection diffusion phenomenon, establish mathematical model of quantitative analysis. Starting from the instantaneous point source, it reflects the scientific thought from micro to macro. Through the integration on time and space, it can be 
extended to a variety of cases, and has good adaptability. When the given system parameters, MATLAB numerical simulation, concentration to obtain at any time and any position, and has certain application value in engineering, can predict the pollution somewhere after a certain period of time, to help people quantitative assessment of pollution and pollution of rational governance.

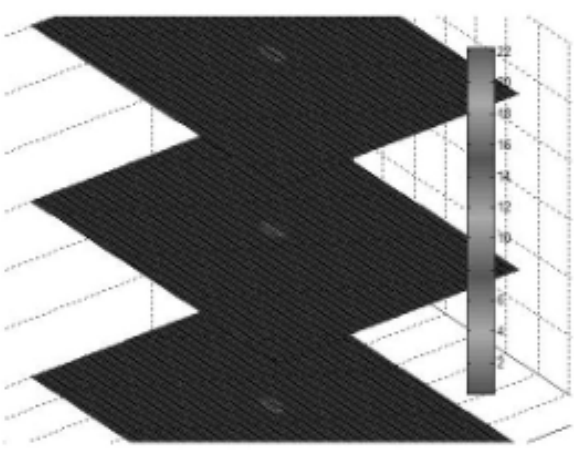

Fig. 3

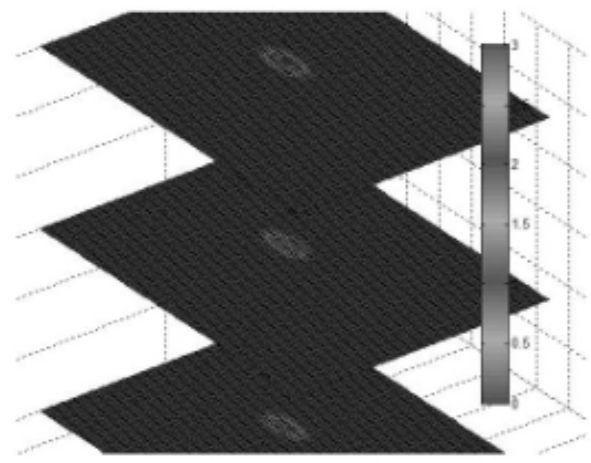

Fig. 5

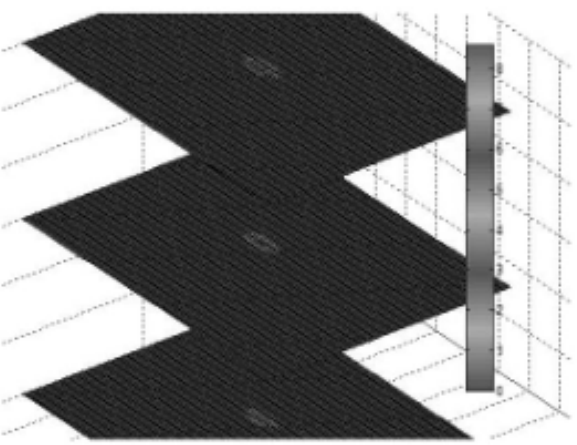

Fig. 4

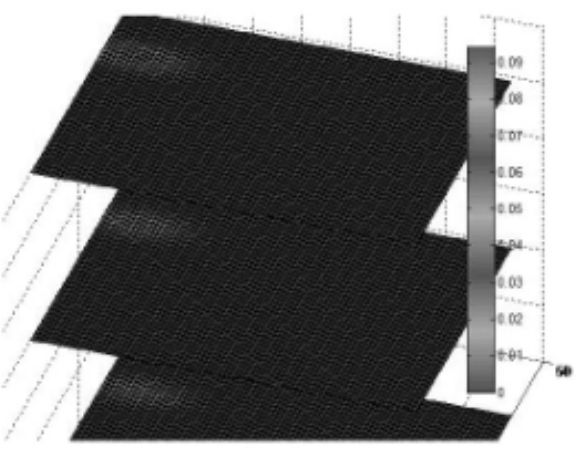

Fig. 6

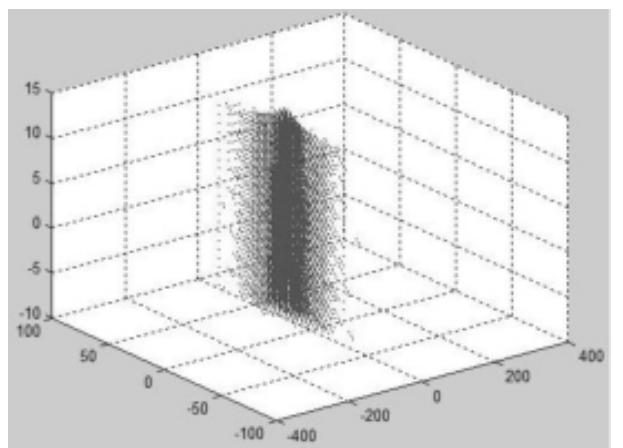

Fig. 7

\section{References}

[1] Q. Y. Jiang, J. X. Xie, J. Ye, "mathematical model," Beijing: Higher Education Press, 2011.

[2] K. S. Liang, "Mathematical physics method," Beijing: Higher Education Press, 2010.

[3] X. X. Li, "Diffusion simulation of sudden pollutants in rivers," Modern Agricultural Science and Technology, vol. 44, no. 6, pp. 196-197, 2015.

[4] J. S. Duan, M. Y. Xu, "Solution of supernormal diffusion model for three-dimensional instantaneous point source," Journal of Shandong University, vol. 37, no. 1, pp. 1-4, 2002.

[5] H. Li, Q. L. Wang, R. Diao, "Dust Diffusion and Visualization of Concentration Distribution in Graphite Processing Workshop," Coal Technology, vol. 36, no. 7, pp. 187-190, 2017.

[6] S. Xue, "MATLAB basic course," Beijing: Tsinghua university press, 2011. 